\title{
Efficacy of physical activity counseling plus sleep restriction therapy on the patients with chronic insomnia
}

\author{
Jihui Wang \\ Guangxia Yin \\ Guanying Li \\ Wenjing Liang \\ Qinling Wei
}

Department of Psychiatry, The Third Affiliated Hospital of Sun Yat-sen University, Guangzhou, People's

Republic of China
Correspondence: Jihui Wang

Department of Psychiatry, The Third Affiliated Hospital of Sun Yat-sen University, No 60, Tianhe Road, Tianhe District, Guangzhou, Guangdong 510630 , People's Republic of China

Tel +862085252190

Fax +86 2085252479

Email jonny928@I26.com
This article was published in the following Dove Press journal:

Neuropsychiatric Disease and Treatment

23 October 2015

Number of times this article has been viewed

Objective: Lack of physical activity (PA) is common in patients with chronic insomnia Studies to increase PA and decrease sedentary behavior in those patients are limited. Therefore, we investigated the efficacy of "PA counseling combined with sleep restriction (SR) therapy (PASR)" vs only SR in the patients with chronic insomnia.

Methods: Seventy-one outpatients were assigned to either PASR $(n=35)$, consisting of four weekly PA counseling sessions based on 5A model (assess, advise, agree, assist, and arrange) + $\mathrm{SR}$, or SR ( $\mathrm{n}=36$ ), consisting of four weekly SR. International Physical Activity Questionnaire (Chinese version) and pedometer-based daily steps were evaluated as the primary endpoints. Insomnia Severity Index, Epworth Sleepiness Scale, Fatigue Scale-14, and Sleep Diary were evaluated as the secondary endpoints.

Results: The results showed that the patients in the PASR group gained more benefits than the SR group in terms of PA level and pedometer-based daily steps (all $P<0.05$ ). Better improvements of the study group were also shown in Epworth Sleepiness Scale, Fatigue Scale-14, and Sleep efficiency (all $P<0.05$ ).

Conclusion: We conclude that PA counseling based on 5A model combined with SR cannot only effectively increase the PA levels but also improve the sleep quality for patients with chronic insomnia.

Keywords: behavioral therapy, physical activity, sleep disorders, sleep restriction, counseling

\section{Introduction}

Insomnia is a common complaint that affects $10 \%-15 \%$ of the general population and carries a heavy burden for both patients and the health care system. ${ }^{1}$ Drug therapy is commonly prescribed for the treatment of chronic insomnia; however, sleeping pills have several known side effects when used for long periods of time. Cognitive behavioral therapy for insomnia (CBT-I), usually including sleep restriction (SR) therapy, stimulus control therapy, and relaxation therapy, is considered the first line treatment for chronic insomnia because of its efficacy and durability. ${ }^{2}$ However, CBT-I is still one of the best kept secrets in the sleep medicine due to some shortages, such as limited CBT-I therapists, high maintenance costs, and low adherence and compliance, which creates the need to develop other non-pharmacological treatment approaches for the management of insomnia.

Among patients with chronic insomnia, physical inactivity, and sedentary lifestyle are very common because of daytime fatigue and somnolence associated with insomnia on the one hand, ${ }^{3}$ on the other hand, physical inactivity can increase the risks of poor 
sleep quality and several other mental health problems. ${ }^{4}$ In recent years, several studies have shown that regular physical activity (PA), especially aerobic exercise training could improve sleep quality and daytime function in patients with chronic insomnia. ${ }^{5-7}$ Therefore, PA intervention aiming to increase the PA level has been proposed as a low-cost, easily accessible, and non-pharmacologic treatment alternative for patients with chronic insomnia. However, most of these studies were conducted under experimental circumstance and based on specific PA prescription. The data about how to increase the PA level in clinical practice is relatively limited. In clinical practice, clinicians often give advice about increasing PA, but studies showed that these low-intensity PA counseling made no overall effect, ${ }^{8}$ more than half of patients receiving such advice were not even able to recall it. ${ }^{9}$ The 5A guidelines, in which clinicians Assess about, Advise about, Agree upon, Assist, and Arrange follow-up concerning patients' behavior change efforts - is a framework for brief PA counseling. ${ }^{10}$ ASSESS is important for the patients to realize that they are in urgent need of change. ADVISE is used to specifically link PA recommendations to a person's own behavioral context and maximize motivation for change. AGREE is helpful for the patient and clinician to come to a common ground and work together on PA goals and strategies. ASSIST is used to offer additional resources, referral options, or practical problem-solving strategies. Finally, ARRANGE is important for behavior change by providing the opportunity to follow-up and adjust the change plan based on the progress the patient has achieved. ${ }^{11}$ Initially developed by the US Cancer Institute as the "4As" for smoking cessation, the 5As have been approved by the US Preventive Services Task Force, ${ }^{11}$ the Canadian Task Force on Preventive Health Care, ${ }^{12}$ and national guidelines in the $\mathrm{UK}^{13}$ as a unifying framework for behavioral counseling. Several studies have shown that the PA counseling based on 5As could increase the PA level of the visitors and improve the communication skill on PA counseling of the clinicians in primary care. ${ }^{14-16}$

Taken together, these results indicate PA as a potential means to improve sleep quality. They also indicate 5As PA counseling as a potential means to increase the PA level of the visitors in primary care settings. However, the efficacy of 5As PA counseling to improve the PA level and sleep quality in patients with chronic insomnia has not been established. Therefore, the aim of this study was to test the hypothesis that 5As PA combined with SR can improve the PA level and self-reported sleep quality in adults with chronic insomnia and is better than only SR.

\section{Materials and methods Study design}

A randomized-controlled trial with assessments at pretreatment, 2-week, and 4-week posttreatment compared the efficacy of PA combined with SR (PASR) vs only SR. PA counseling and SR are both four weekly manual-based intervention. The ethics committee of Sun Yat-sen University reviewed and approved the study protocol. All the participants provided written informed consent.

\section{Participants}

Participants were recruited from the outpatient clinics in the psychology department of the Third Affiliated Hospital of Sun Yat-sen University between January 2014 and December 2014. Inclusion criteria: a) met research diagnostic criteria for insomnia disorder, ${ }^{17}$ which specify a sleep complaint lasting for at least 6 months, a mean total wake time (sleep onset + wake after onset) of more than 60 minutes per night during a screening week of sleep-diary monitoring, adequate opportunity, and circumstances for sleep, and significant distress or daytime impairment. Insomnia Severity Index (ISI) scale $\geq 10$; b) age 18-60 years old; c) independent in activities of daily living and being able to walk for 10 minutes without aids; and d) participants who were using sedative medications were asked to discontinue these sedatives gradually 2 weeks before participating in the study. Anti-depressants should be continued, and the dosage was not changed during the study.

Exclusion criteria: a) history of $D S M-I V$ criteria for any major psychiatric disorder, including mania or alcohol or substance abuse; b) unstable or serious medical conditions or cardiopulmonary disease that contraindicate exercise; c) untreated other sleep disorder; d) idea and/or behavior of suicide recently; e) night shift; and f) experience of SR or PA counseling.

\section{Procedure}

Potential subjects underwent a brief face-to-face screening, of which 158 subjects passed this stage and completed a structured interview and a sleep-diary monitoring for 2 weeks. At last, 71 volunteers qualified, underwent pretreatment assessment, received sleep hygiene ( $\mathrm{SH}$ ) education, and subsequently were allocated to PASR (35 participants) or SR (36 participants). One clinical psychologist with 10 years experience of conducting behavioral therapy delivered PA counseling and SR treatments. Another psychologist who was blind to the therapy conditions served as questionnaires assessor. 


\section{Randomization}

Participants were randomly allocated to their respective treatment groups, using a randomization ratio of $1: 1$, by drawing lots. Allocation concealment was achieved by drawing cards from an opaque box. The assessor of the study questionnaires and the assistants who recorded the data were blinded to group allocation.

\section{Treatments}

\section{SR therapy}

In the first session, total sleep time (TST) was estimated according to the sleep diary, and time-in-bed (TIB) (not $<5$ hours) were set as: TIB $=\mathrm{TST}+30$ minutes. In the following sessions, the TIB of the next week would be set according to the sleep efficacy (SE), $\mathrm{SE}=\mathrm{TST} / \mathrm{TIB}$. If SE $>90 \%$, the TIB would be increased by 15 minutes; while if SE $<85 \%$, the TIB would be reduced by 15 minutes. For those $85 \%<$ TIB $<90 \%$, the TIB would be kept unchanged.

\section{Sleep hygiene education}

Therapist presented the basic four SH recommendations, including avoiding caffeine or alcohol before bedtime, exercising regularly, avoiding being hungry, or eating too much before bed and maintaining a comfortable sleeping environment.

\section{PA counseling}

A four weekly PA counseling treatment based on the 5A model was applied to the PASR group.

\section{Sessions content}

\section{Session I (approximately 60 minutes)}

Session 1 is used to a) assess current PA (FITT: frequency, intensity, type, and time), contraindications to PA, readiness for change, patient-oriented benefits, social support, willingness and self-efficacy to make a change; b) advise on personal health benefits and FITT principles with the guidelines in mind which is at least 30 minutes of accumulated moderate-intensity PA (ie, walking fast [3-4 miles per hour] or the equivalent) on 5 or more days of the week. Provide a tailored message and specific advice according to the patient's stage of change; c) agree on goals and develop action plans which are concrete and defined in behavioral terms rather than outcomes. Encourage the patient to actively engage in goal setting; $d$ ) assist the patient to carry out the action plan with a written PA prescription and self-monitoring calendar; and e) arrange for follow-up assessment and visit.

\section{Sessions 2 and 3 (approximately 15 minutes, respectively)}

The follow-up second and third sessions are used to review progress, provide ongoing support, adjust PA prescription, overcome identified barriers, and enhance adherence to action plan.

\section{Session 4 (approximately 15 minutes)}

Session 4 is the last follow-up visit which aims to review the whole progress of counseling, continue the adjustment of PA prescription, discuss potential difficulties, and instruct the patient in relapse-prevention strategies. ${ }^{10,11}$

\section{Measures}

\section{International PA Questionnaire}

International PA Questionnaire (IPAQ) (long-form and Chinese version) with good reliability and validity was used to evaluate the PA levels during the past week ${ }^{18}$ Cronbach's alpha in this study was 0.83 . IPAQ-long form is designed to measure cross-national PA in adults and considers workrelated PA, transport PA, domestic and gardening PA, and leisure PA. It asks respondents to describe their time spent on walking, moderate PA, and vigorous PA within each domain. Energy expenditure was evaluated by Metabolic Equivalent (MET). One MET equivalent to the energy consumed by a normal resting adult. IPAQ data were used to estimate total weekly PA by weighting the reported minutes per week within each activity category by a MET energy expenditure estimate assigned to each category of activity. ${ }^{19}$ The weighted MET-minutes per week (MET-min/week) were calculated as duration * frequency per week * MET intensity, which were summed across activity domains to produce a weighted estimate of total PA from all reported activities per week (MET-min/week). The main outcome examined was the difference between the two groups with regard to the changes in the grading of PA levels. The grading of the PA levels was: a) high PA level was defined as "vigorous activity on $\geq 3$ days achieving $\geq 1,500$ MET-min/week, or $\geq 5$ days of walking, moderate and/or vigorous activities achieving $\geq 3,000$ MET-min/week"; b) Moderate PA level was defined as " $\geq 3$ days with $\geq 20$ minutes of vigorous activity, or $\geq 5$ days with $\geq 30$ minutes of moderate activity and/or walking, or $\geq 5$ days of walking, moderate and/or vigorous activities achieving $\geq 600$ MET-min/week"; and 
c) Low PA level was defined as "any PA level not meeting the criteria for moderate or high PA level". ${ }^{20}$

\section{A 6-minute walk test}

The 6-minute walk test is a submaximal test showing the walking distance in 6 minutes (6MWD) which the patient can quickly walk on a flat, hard surface in a period of 6 minutes. It reflects the patient's level of physical fitness in daily life. ${ }^{21}$

\section{Pedometer-based daily steps}

An Omron HJ-203-ED pedometer with good validity and reliability was used to measure daily steps of the participants for 7 consecutive days in the 1 st and 4 th week. ${ }^{22}$ Participants were instructed to wear the pedometer around the neck, given that the least amount of error was observed for this wearing position. Participants not providing $\geq 5$ days of $\geq 540$ minutes ( 9 hours) per day of data were asked to rewear the pedometer. The mean number of steps was calculated by the ratio between the sum of the daily totals and the number of days the pedometer was used.

\section{The Insomnia Severity Index}

ISI was used to evaluate the insomnia severity of the subjects. There are totally seven items, each scored from 0 to 4 $(0=$ not at all, $4=$ extremely). Total scores ranged from 0 to $28 .{ }^{23}$ Cronbach's alpha in this study was 0.81 .

\section{Epworth sleepiness Scale}

Epworth sleepiness Scale (ESS) was used to evaluate the daytime somnolence. The Scale included eight items, with each score from 0 to 3 ( $0=$ never, $4=$ high chance). Total scores ranged from 0 to $24 .{ }^{24}$ Cronbach's alpha in this study was 0.79 .

\section{Fatigue Scale- 14}

Fatigue Scale-14 (FS-14) was used to evaluate daytime fatigue symptoms. The scale included 14 items, with each scores $0-1$. Total scores ranged from 0 to $14 .{ }^{25}$ Cronbach's alpha in this study was 0.75 .

\section{Sleep diary}

Each patient was required to complete a daily sleep diary. The variables extracted from the sleep diaries were TIB, TST, wake after sleep onset, sleep onset latency, and sleep efficiency $(\mathrm{SE}=(\mathrm{TST} / \mathrm{TIB}) \times 100)$.

\section{Treatment compliance}

At the end of the treatment, all the patients were asked to complete a brief questionnaire to assess SR treatment adherence. They were asked how many days per week they enacted each of two core elements of the SR recommendations (ie, adherence to TIB prescription and standard rise time).

\section{Statistical analysis}

SPSS16.0 was introduced for statistical analysis. PA levels were graded into "low, moderate, and high", with assigning values as 1,2, and 3, respectively. The changes in the PA grades after the treatment were calculated, and the difference between the two groups was then tested by Mann-Whitney $U$-test. Continuous variables (PA energy expenditure, pedometer-based daily steps, 6MWD, and sleep-related indices) were evaluated by repeated-measure analyses of variance (ANOVA). Two separate $F$-tests were performed for the two groups individually at first. Then, the difference between the two groups was evaluated, with factors as a group, time (pretreatment or posttreatment) and group $\times$ time interaction. Age, body mass index (BMI), and medication (with assigning values as "use $=0$, no use $=1$ ") were added to all equations as covariates. Fisher exact statistics was applied to unordered categorical variables, with ordered categorical variables compared with ridit analysis. In cases of premature study termination, analysis was conducted using "last observation carried forward". Statistical significance was set at $\alpha=0.05$ (two-tailed).

\section{Results}

\section{Clinical features of the participants}

For the PASR group, age was (22-58) years old, with the course of insomnia (0.5-12) years; BMI (18.05-28.66) kg/m², ISI (11-19), ten cases being primary cases, 17 cases being co-morbid with general anxiety disorder, eight cases being co-morbid with major depression. For the SR group, age was (28-58) years, with the course of insomnia (0.5-12) years, BMI (18.05-22.66) kg/m², ISI (10-19), nine cases being primary insomnia, 17 cases being co-morbid with general anxiety disorder and ten cases being co-morbid with major depression (Table 1).

\section{Withdraw cases}

Two cases withdrew from the PASR group. One was due to muscle injury, and the other was due to the need to use sedative medication. Three cases withdrew from the SR group, one was due to moving to another city, and two cases were due to the use of sedative medication. There was no statistical significance in the withdrawal rate between the two groups $\left(\chi^{2}=0.186, P=0.666\right)$.

\section{SR treatment compliance}

The compliance to "standard rise time" was $(5.15 \pm 1.73)$ days/ week in the PASR group and (5.36 \pm 1.49$)$ days/week in the SR group. The difference was not statistical significant $(P>0.05)$. The compliance to "TIB prescription” was (6.05 \pm 1.23$)$ days/week 
Table I Baseline demographic and clinical characteristics

\begin{tabular}{|c|c|c|c|c|}
\hline Variables & Study group $(n=35)$ & Control group $(n=36)$ & Statistical values & $P$-value \\
\hline Age (Mean $\pm S D$, years) & $38.7 I \pm 9.93$ & $41.77 \pm 7.65$ & $Z=1.442$ & 0.154 \\
\hline $\operatorname{Sex}(n)$ & & & $\chi^{2}=0.113$ & 0.737 \\
\hline Male & 13 & 12 & & \\
\hline Female & 22 & 24 & & \\
\hline Course of insomnia (Mean $\pm S D$, years) & $3.47 \pm 2.74$ & $3.67 \pm 3.18$ & $Z=0.042$ & 0.967 \\
\hline BMI $\left(\right.$ Mean $\left.\pm S D, k g / \mathrm{m}^{2}\right)$ & $22.60 \pm 2.69$ & $21.91 \pm 2.85$ & $Z=0.910$ & 0.363 \\
\hline Type of insomnia (n) & & & $\chi^{2}=0.115$ & 0.734 \\
\hline Primary insomnia & 10 & 9 & & \\
\hline Co-morbid insomnia & 25 & 27 & & \\
\hline PA levels at week $0(n)$ & & & $F=0.178$ & 0.674 \\
\hline Low & 21 & 24 & & \\
\hline Moderate & 12 & 9 & & \\
\hline High & 2 & 3 & & \\
\hline Anti-depressants (n) & & & $\chi^{2}=0.351$ & 0.553 \\
\hline Yes & 23 & 26 & & \\
\hline No & 12 & 10 & & \\
\hline Type of anti-depressants ( $n$ ) & & & $\chi^{2}=0.344$ & 0.842 \\
\hline SSRI & 15 & 18 & & \\
\hline SNRI & 3 & 4 & & \\
\hline NaSSA & 5 & 4 & & \\
\hline
\end{tabular}

Abbreviations: BMI, body mass index; PA, physical activity; SSRI, selective serotonin reuptake inhibitor; SNRI, selective noradrenalin reuptake inhibitor; NaSSA, noradrenergic and specific serotonergic antidepressant.

in the PASR group and (5.92 \pm 1.11$)$ days/week in the SR group. The difference was not statistical significant $(P>0.05)$.

\section{Grading of PA levels}

At the end of week 4, there were 10,20, and 5 cases reported as low, moderate, and high PA level, respectively, in the PASR group and 20,13, and 3 cases, respectively, in the SR group. The subjects in the PASR group made more improvements than those in the SR group $(Z=2.032, P=0.016)$.

\section{Energy expenditure}

The ANOVA showed significant treatment time main effects on transportation METs, leisure-time METs, "moderate + vigorous" PA duration, time spending on sitting and sleeping for both groups, as well as a significant treatment group $\times$ time interaction effects on transportation METs $(F=7.658, P=0.006)$, leisure-time METs $(F=4.586, P=0.048)$, “moderate + vigorous" PA METs $(F=12.502, P<0.001)$, "moderate + vigorous" PA duration $(F=6.554, P=0.015)$ and time spending on sitting $(F=11.584, P<0.001)$. The effect size was 0.43 for transportation METs, 0.43 for leisure-time METs, 0.84 for "moderate + vigorous" PA METs, 0.97 for "moderate + vigorous" PA duration and 1.10 for time spending on sitting (Table 2).

\section{Pedometer-based daily step counts}

Daily step counts of the PASR group increased from $(7,854.3 \pm 2,185.6)$ steps at baseline to $(11,045.6 \pm 3,742.5)$ steps at the end of week 4, while daily step counts of the SR group increased from $(7,694.4 \pm 2,358.6)$ steps at baseline to $(8,654.62 \pm 3,325.6)$ steps at the end of week 4 . The ANOVA showed a significant time main effects in both groups, and a significant treatment group $\times$ time interaction effects $(F=7.256, P=0.007)$. The effect size was 0.72 .

\section{6-minute walk distance}

At baseline, the 6MWD was $(500.46 \pm 81.59) \mathrm{m}$ in the PASR group and (511.80 \pm 66.38$) \mathrm{m}$ in the SR group; while at the end of week 4, the 6MWD was $(544.97 \pm 69.43) \mathrm{m}$ in the PASR group and (533.49 \pm 60.95$) \mathrm{m}$ in the SR group. The ANOVA showed a significant time main effects in both groups, as well as a significant treatment group $\times$ time interaction effects $(F=9.245, P=0.004)$. The effect size was 0.61 .

\section{Sleep related index}

The ANOVA showed a significant treatment time main effects on ISI, ESS, FS-14, sleep onset latency, SE, and wake after sleep onset, as well as significant treatment group $\times$ time interaction effects on $\operatorname{ESS}(F=8.135, P=0.006)$, FS-14 ( $F=5.256, P=0.032), \operatorname{SE}(F=5.426, P=0.024)$. The effect size was 0.72 for ESS, 0.62 for FS-14 and 0.63 for SE (Table 3).

\section{Discussion}

The results of this study supported the efficacy of 5As PA counseling combined with SR in patients with chronic insomnia. 

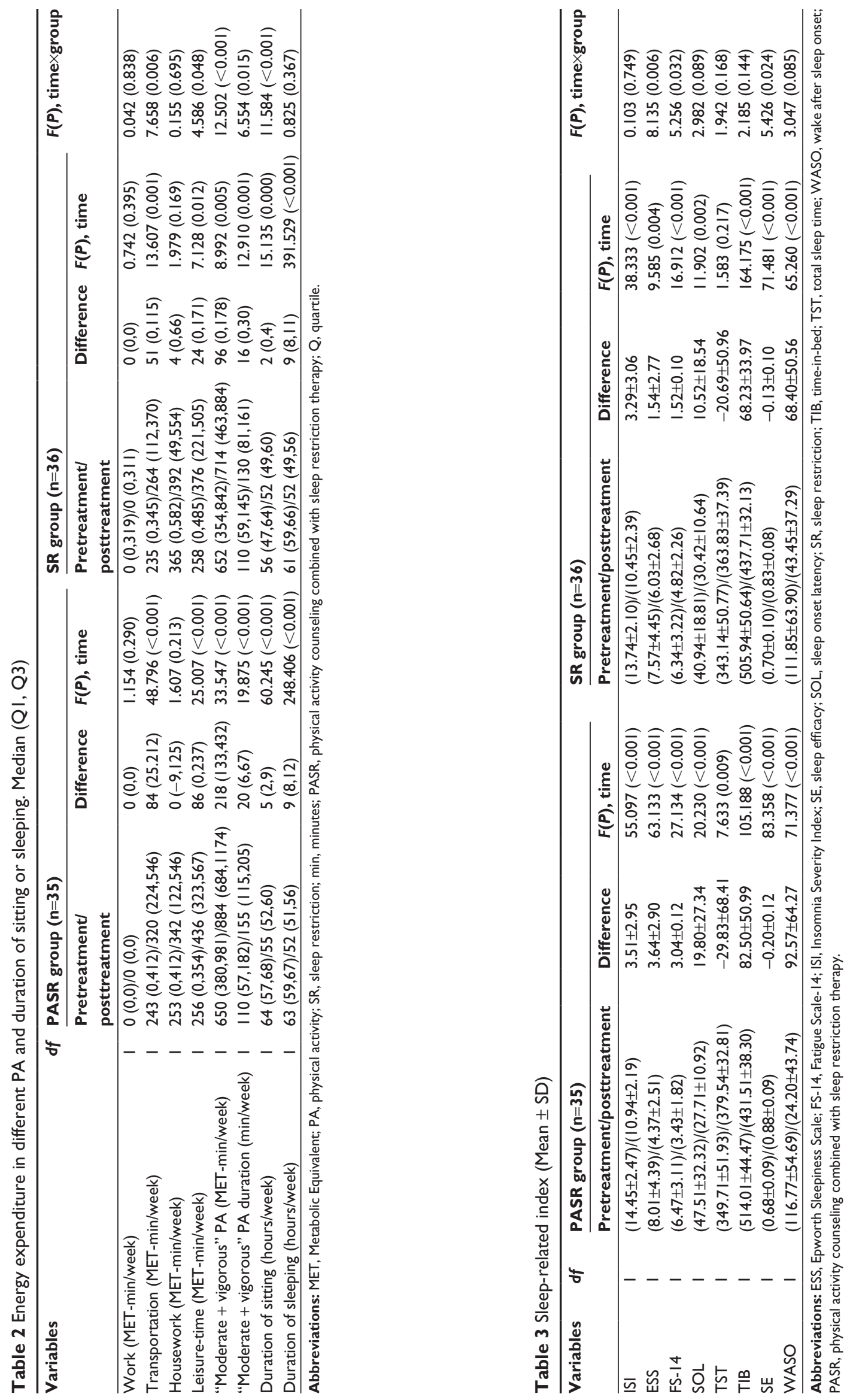
The combined treatment was more effective than only SR therapy to improve the PA levels and sleep quality.

Setting the "moderate intensity PA for 30 minutes a day, at least 5 days a week" as a recommended standard, ${ }^{26}$ approximately half of US adults cannot reach the level recommended as beneficial to health. ${ }^{27}$ Experts of the IPAQ recommended a similar standard as above. Using the IPAQ standard, some Chinese studies claimed that the $89.4 \%$ and $88.8 \%$ residents in Jiangsu Province and Shanghai Urban Area, respectively, have adequate PA. ${ }^{28,29}$ The reason for this may be that the Chinese usually spend more time on transportation, working, and housework, that requires more physical activities. ${ }^{30}$ The cases involved in this study had a worse level of PA than the normal residents reported in the studies mentioned above. At week 0, there were only $35.7 \%$ (25/70) subjects at adequate PA level. The reason for this may be insomnia, but may also be related to the geographic and economic factors. ${ }^{31}$

The subjects of both groups showed improvement in sleeping quality, daytime somnolence, and fatigue. However, the subjects in the SR group did not achieve such significant improvement in grading of PA levels as those in the PASR group. Differences of the treatment effectiveness between two groups imply the following two points: 1) although insomnia related daytime somnolence and fatigue may be responsible for the reduction of PA in chronic insomnia patients, improving the sleep conditions may not necessarily lead to the improvement of PA. It may be because of lacking awareness on the importance of PA, or lacking confidence to increase their PA, or having no idea of how to increase their PA. ${ }^{32}$ We suggest, therefore, paying extra attention to patients' conditions of PA when treating chronic insomnia and giving standard clinical counseling. 2) PA counseling in low intensity may be not enough to improve the PA levels. USPSTF (2006) classifies the intensity of PA counseling into three grades and suggests that to make the counseling effective, and the counseling intensity should be at least moderate. ${ }^{33}$ Some studies claimed that low-intensity counseling may be also effective, but they were focused on normal population ${ }^{34}$ or population with low PA level. ${ }^{35}$ In our study, the SH education which included the advice of "carry out PA regularly" was low-intensity counseling. It turned out to be not effective enough to improve the PA levels. On the other hand, the counseling based on the 5A model had some advantages as follows: It was in moderate intensity, and the patients were more involved in the counseling and more individualized. And more specific treatment plan were set based on detailed evaluations, and the plan could be adjusted immediately and better fit the subjects based on the feedback from the subjects. All these traits made the effectiveness of the treatment better. Although we did not limit the PA level at week 0 strictly, evidence has shown that more PA appeared to provide more benefit. ${ }^{10}$

Although both groups showed improvement in multiple indices of the sleeping conditions, the subjects in the PASR group had a $20 \%$ improvement in SE, better than the SR group (13\%), consistent with several previous studies. ${ }^{36,37}$ The better improvements in our study may indicate that PA counseling can enhance the efficiency of the behavioral therapy for insomnia. Although the change of SE might be the direct effect of SR instead of the improvement of sleep quality, the decrease of daytime sleepiness and fatigue confirmed the improvement of sleep quality. ${ }^{38}$ The improvement of sleep quality might be partially due to the increase of the PA level because a number of studies have shown that with increased PA, insomnia patients can improve not only their sleeping quality, but also the daytime function and the quality of life. Most of these studies, however, were carried out under specific experimental condition. ${ }^{5,39,40}$ In fact, incidental and lifestyle-embedded PA can also help sleep. ${ }^{41}$ Therefore, the PA we defined included not only physical exercises but also daily activities in our study.

There were some limitations in our study. Firstly, the results might be influenced because some subjects continued to use anti-depressants during the study. Secondly, the observation duration was relatively short in our study. Despite the above limitations, our study still has some clinical significance. Firstly, co-morbid insomnia is very common and medication together with behavioral therapy is a common measure in clinical practice. Furthermore, the subjects in our study were in stable stage. Medications were similar in two groups at week 0 , and the dosage was not changed during the study. These measures helped to reduce the impact due to the medications. We believe that long-term follow-up interview in the future studies is warranted.

\section{Disclosure}

The authors report no conflicts of interest in this work.

\section{References}

1. Ohayon MM. Epidemiology of insomnia: what we know and what we still need to learn. Sleep Med Rev. 2002;6(2):97-111.

2. Hood HK, Rogojanski J, Moss TG. Cognitive-behavioral therapy for chronic insomnia. Curr Treat Options Neurol. 2014;16(12):321.

3. Chasens ER, Yang K. Insomnia and physical activity in adults with prediabetes. Clin Nurs Res. 2012;21(3):294-308.

4. Wu X, Tao S, Zhang Y, Zhang S, Tao F. Low physical activity and high screen time can increase the risks of mental health problems and poor sleep quality among Chinese college students. PLoS One. 2015; 10(3):e0119607. 
5. Passos GS, Poyares D, Santana MG, et al. Effects of moderate aerobic exercise training on chronic primary insomnia. Sleep Med. 2011; 12(10):1018-1027.

6. Hartescu I, Morgan K, Stevinson CD. Increased physical activity improves sleep and mood outcomes in inactive people with insomnia: a randomized controlled trial. J Sleep Res. 2015;24(5):526-534.

7. Reid KJ, Baron KG, Lu B, Naylor E, Wolfe L, Zee PC. Aerobic exercise improves self-reported sleep and quality of life in older adults with insomnia. Sleep Med. 2010;11(9):934-940.

8. Lin JS, O'Connor E, Whitlock EP, Beil TL. Behavioral counseling to promote physical activity and a healthful diet to prevent cardiovascular disease in adults: a systematic review for the U.S. Preventive Services Task Force. 2010;153(11):736-750.

9. Flocke SA, Stange KC. Direct observation and patient recall of health behavior advice. Prev Med. 2004;38(3):343-349.

10. Beaulac J, Carlson A, Boyd RJ. Counseling on physical activity to promote mental health: practical guidelines for family physicians. Can Fam Physician. 2011;57(4):399-401.

11. Whitlock EP, Orleans CT, Pender N, Allan J. Evaluating primary care behavioral counseling interventions: an evidence-based approach. Am J Prev Med. 2002;22(4):267-284.

12. Elford RW, MacMillan HL, Wathen CN. Canadian Task Force on Preventive Health Care. Counseling for risky health habits: a conceptual framework for primary care practitioners. London, ON: CTFPHC Technical Support, Canadian Task Force; 2001.

13. Khan KM, Weiler R, Blair SN. Prescribing exercise in primary care. BMJ. 2011;343:d4141.

14. Carroll JK, Fiscella K, Epstein RM, Sanders MR, Williams GC. A 5A's communication intervention to promote physical activity in underservedpopulations. BMC Health Serv Res. 2012;12:374.

15. Carroll JK, Antognoli E, Flocke SA. Evaluation of physical activity counseling in primary care using direct observation of the 5As. Ann Fam Med. 2011;9(5):416-422.

16. Carroll JK, Winters PC, Sanders MR, Decker F, Ngo T, Sciamanna CN. Clinician-targeted intervention and patient-reported counseling on physical activity. Prev Chronic Dis. 2014;11:E89.

17. Sleep disorder research group of the neurology chapter of Chinese Medical Association. Guidelines of diagnosis and treatment of insomnia in adult Chinese population. Chin J Neurol. 2012;45:534-540.

18. Qu NN, Li KJ. Study on the reliability and validity of international physical activity questionnaire (Chinese Vision, IPAQ), Chin J Epidemiol. 2004;25(3):265-268.

19. Bassett DR Jr. International physical activity questionnaire: 12-country reliability and validity. Med Sci Sports Exerc. 2003;35(8):1396.

20. Fan M, Lyu J, He P. Chinese guidelines for data processing and analysis concerning the International Physical Activity Questionnaire. Zhonghua Liu Xing Bing Xue Za Zhi. 2014;35(8):961-964.

21. American Thoracic Society. ATS statement: guidelines for the sixminute walk test. Am J Respir Crit Care Med. 2002;(166):111-117.

22. De Cocker KA, De Meyer J, De Bourdeaudhuij IM, Cardon GM. Nontraditional wearing positions of pedometers: validity and reliability of the Omron HJ-203-ED pedometer under controlled and free-living conditions. J Sci Med Sport. 2012;15(5):418-424.

23. Bastien CH, Vallieres A, Morin CM. Validation of the Insomnia Severity Index as an outcome measure for insomnia research. Sleep Med. 2001;2(4):297-307.
24. Johns MW. A new method for measuring daytime sleepiness: the Epworth sleepiness scale. Sleep. 1991;14(6):540-545.

25. Chalder T, Berelowitz G, Pawlikowska T, et al. Development of a fatigue scale. J Psychosom Res. 1993;37(2):147-153.

26. Jacobson DM, Strohecker L, Compton MT, Katz DL. Physical activity counseling in the adult primary care setting: position statement of the American College of Preventive Medicine. Am J Prev Med. 2005; 29(2):158-162.

27. Centers for Disease Control and Prevention. Adult participation in recommended levels of physical activity-United States, 2001 and 2003. MMWR Morb Mortal Wkly Rep. 2005;54(47):1208-1212.

28. Liu CJ, Zhang ZC, Li S, Cao L. A research on people's physical activity level in the three cities of Jiangsu province. J Nanjing Inst Phys Educ. 2012;26(3):43-47.

29. Li Y, Li WT, Fan BH, Fu H. The investigation of the residents' physical activities in the central area of Shanghai. Chin J Ind Hyg Occup Dis. 2004;22(6):458-460.

30. Luan DC, Ma GA. The recommended amount and evaluation criterion for physical activity. Foreign Med Sci Hyg. 2006;33(3):161-165.

31. Zhang YF, Jiang CM, Cai R, Wu DM, Li JJ. Regional characteristics of the national physical activity level in China. China Sport Sci. 2012;32(9):3-10,22.

32. Trost SG, Owen N, Bauman AE, Sallis JF, Brown W. Correlates of adults' participation in physical activity: review and update. $\mathrm{Med} \mathrm{Sci}$ Sports Exerc. 2002;(34):1996-2001.

33. Moyer VA; U.S. Preventive Services Task Force. Behavioral counseling interventions to promote a healthful diet and physical activity for cardiovascular disease prevention in adults: U.S. Preventive Services Task Force recommendation statement. Ann Intern Med. 2012; 157(5):367-371.

34. Marcus BH, Napolitano MA, King AC, et al. Telephone versus print delivery of an individualized motivationally tailored physical activity intervention: project STRIDE. Health Psychol. 2007;26(4):401-409.

35. Grandes G, Sanchez A, Sanchez-Pinilla RO, et al. Effectiveness of physical activity advice and prescription by physicians in routine primary care: a cluster randomized trial. Arch Intern Med. 2009; 169(7):694-701.

36. Taylor DJ, Schmidt-Nowara W, Jessop CA, Aheam J. Sleep restriction therapy and hypnotic withdrawal versus sleep hygiene education in hypnotic using patients with insomnia. J Clin Sleep Med. 2010; 6(2):169-175.

37. Buysse DJ, Germain A, Moul DE, et al. Efficacy of brief behavioral treatment for chronic insomnia in older adults. Arch Intern Med. 2011; 171(10):887-889.

38. Passos GS, Poyares DL, Santana MG, Tufik S, Mello MT. Is exercise an alternative treatment for chronic insomnia? Clinics (Sao Paulo). 2012; 67(6):653-660.

39. Reid KJ, Baron KG, Lu B, Naylor E, Wolfe L, Zee PC. Aerobic exercise improves self-reported sleep and quality of life in older adults with insomnia. Sleep Med. 2010;11(9):934-940.

40. Passos GS, Poyares D, Santana MG, Garbuio SA, Tufik S, Mello MT. Effect of acute physical exercise on patients with chronic primary insomnia. J Clin Sleep Med. 2010;6(3):270-275.

41. Tremblay MS, Esliger DW, Tremblay A, Colley R. Incidental movement, lifestyle-embedded activity and sleep: new frontiers in physical activity assessment. Can J Public Health. 2007;98(Suppl 2):S208-S217.
Neuropsychiatric Disease and Treatment

\section{Publish your work in this journal}

Neuropsychiatric Disease and Treatment is an international, peerreviewed journal of clinical therapeutics and pharmacology focusing on concise rapid reporting of clinical or pre-clinical studies on a range of neuropsychiatric and neurological disorders. This journal is indexed on PubMed Central, the 'PsycINFO' database and CAS,

\section{Dovepress}

and is the official journal of The International Neuropsychiatric Association (INA). The manuscript management system is completely online and includes a very quick and fair peer-review system, which is all easy to use. Visit http://www.dovepress.com/testimonials.php to read real quotes from published authors. 